\title{
Association between changes in salivary microbiota with glucose levels during pregnancy - findings from a pilot study
}

\section{Xin Zhang}

Peking Union Medical College Hospital Eastern Branch: Peking Union Medical College Hospital

\section{Pei Wang}

Peking Union Medical College Hospital Eastern Branch: Peking Union Medical College Hospital

\section{Liangkun Ma}

Peking Union Medical College Hospital Eastern Branch: Peking Union Medical College Hospital

\section{Rongjun Guo}

Bioinformatics Institute

\section{Yongjing Zhang}

Bioinformatics Institute

\section{Peng Wang}

Bioinformatics Institute

Juntao Liu

Peking Union Medical College Hospital Eastern Branch: Peking Union Medical College Hospital Jizhi Zhao ( $\nabla$ zhaojizhi@126.com )

Peking Union Medical College Hospital Eastern Branch: Peking Union Medical College Hospital

\section{Research article}

Keywords: salivary microbiota, oral microbiota, pregnancy, fasting blood glucose, gestational diabetes mellitus

Posted Date: December 4th, 2020

DOl: https://doi.org/10.21203/rs.3.rs-119072/v1

License: (c) (i) This work is licensed under a Creative Commons Attribution 4.0 International License. Read Full License 


\section{Abstract}

Background: Microbial shifts that correspond to host variations during pregnancy are vital in health maintenance. Significant changes have been reported in the oral microbiota of pregnant women when compared with nonpregnant women, but little is known about the dynamic shifts in oral microbiota during the pregnancy course.

Methods : In this study, changes in salivary microbiota in 81 healthy pregnant women throughout the early stage (G1: 9-14 weeks), middle stage (G2: 21-28 weeks), and late stage (G3: 31-38 weeks) were investigated with $16 \mathrm{~S}$ rRNA sequencing techniques. Correlations between salivary microbiota and maternal characteristics, including fasting blood glucose (FBG) levels, were evaluated.

Results : Alpha diversity indexes were stable throughout pregnancy, but significant changes were found in beta diversity measured by weighted and unweighted UniFrac distances. Fourteen dominant trimesterspecific taxa were identified using the LEfSe method, including Bacteroidetes in G1, Proteobacteria in G2 and Firmicutes in G3 at the phylum level. Tax4Fun prediction analysis revealed significant changes in Genetic Information Processing, Environmental Information Processing, Unclassified and Human Diseases in G2 and in Metabolism in G3 when compared to G1. Significant correlations were found between FBG levels and microbial composition, and these correlations were independent of gestational diabetes mellitus (GDM) status.

Conclusion : Within the limitations of this study, the dynamic changes in salivary microbiota during pregnancy were characterized, and beyond pregnancy, FBG was also involved in shaping the salivary microbiota.

\section{Full Text}

This preprint is available for download as a PDF.

\section{Figures}




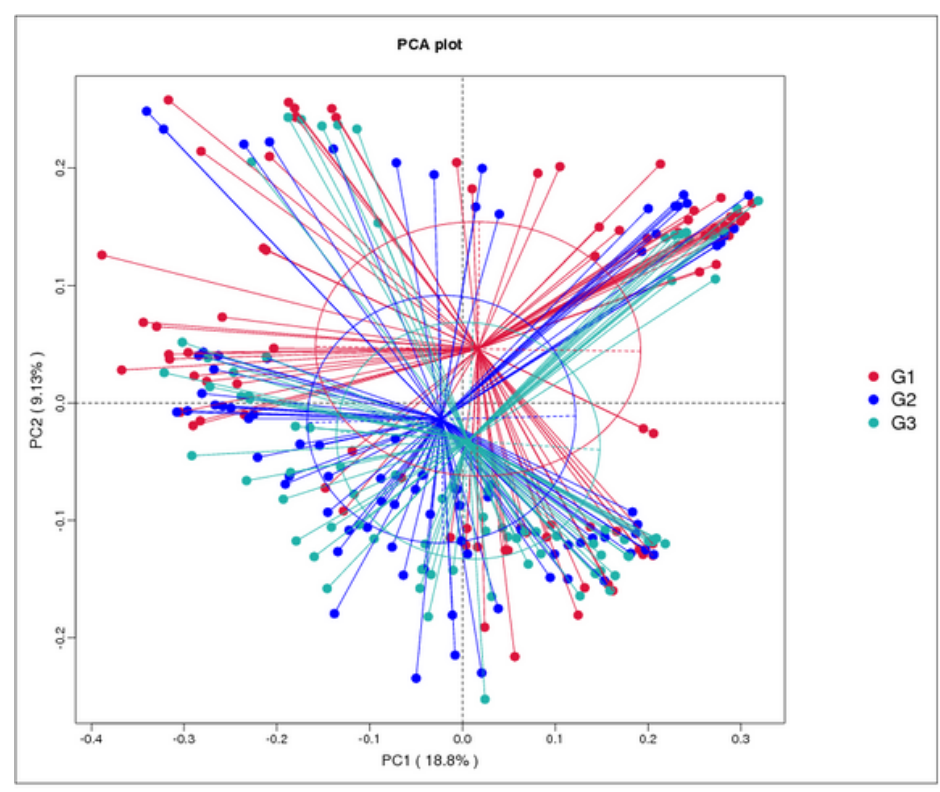

A

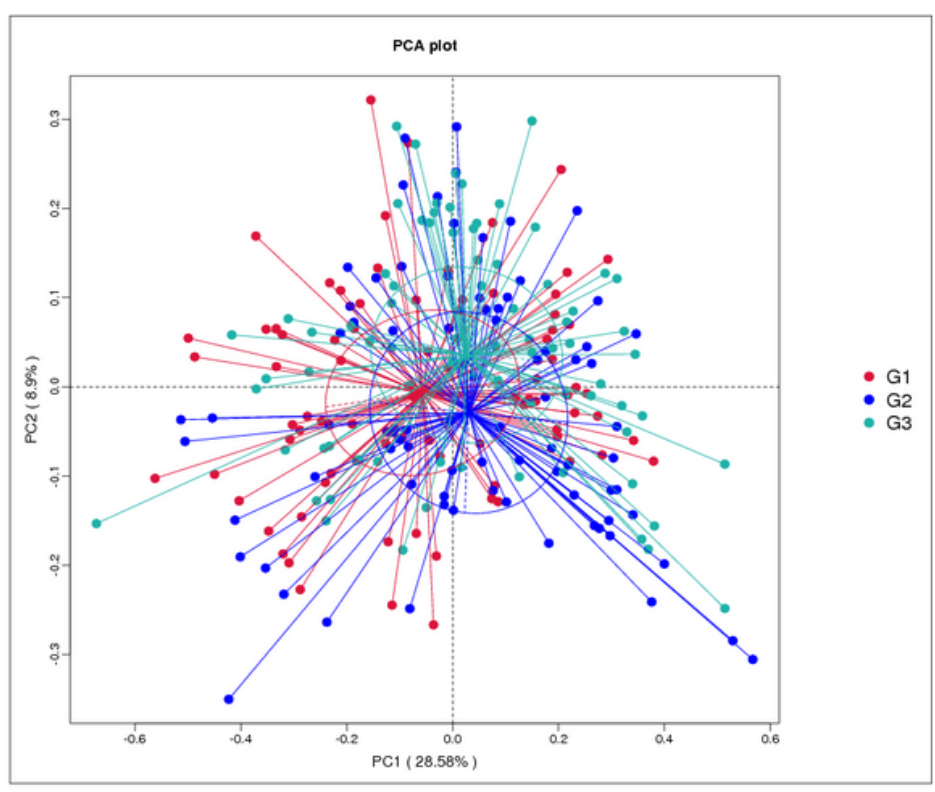

B

\section{Figure 1}

Principal components 1 and 2 based unweighted (A) and weighted (B) principal coordinate analysis (PCoA) plots. G1, G2, and G3 stand for early, middle and late pregnancy, respectively.
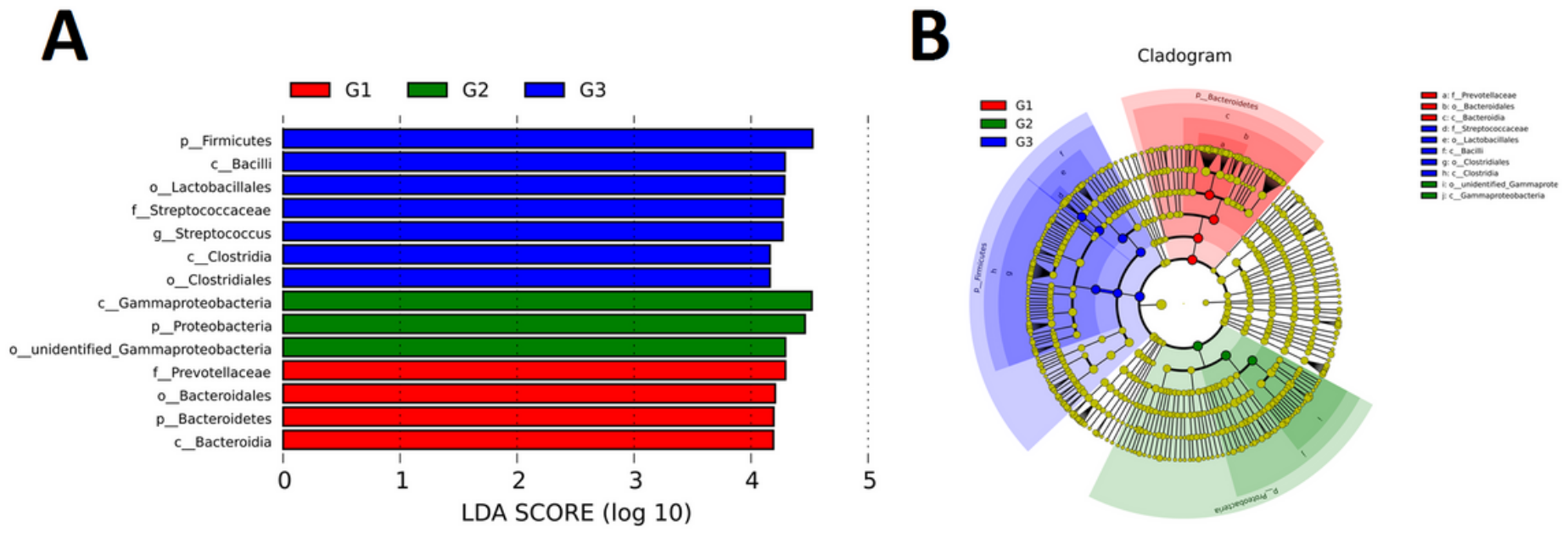

Figure 2

The differentially expressed bacteria identified by LEfSe analysis. Histogram of the linear discriminant analysis (LDA) scores (A). Cladogram for taxonomic representation (B). G1, G2, and G3 stand for early, middle and late pregnancy, respectively. 
A

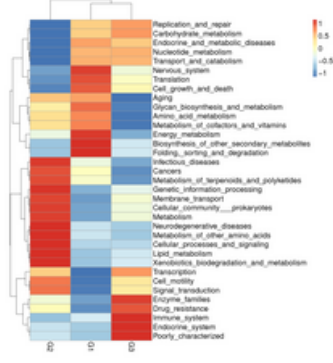

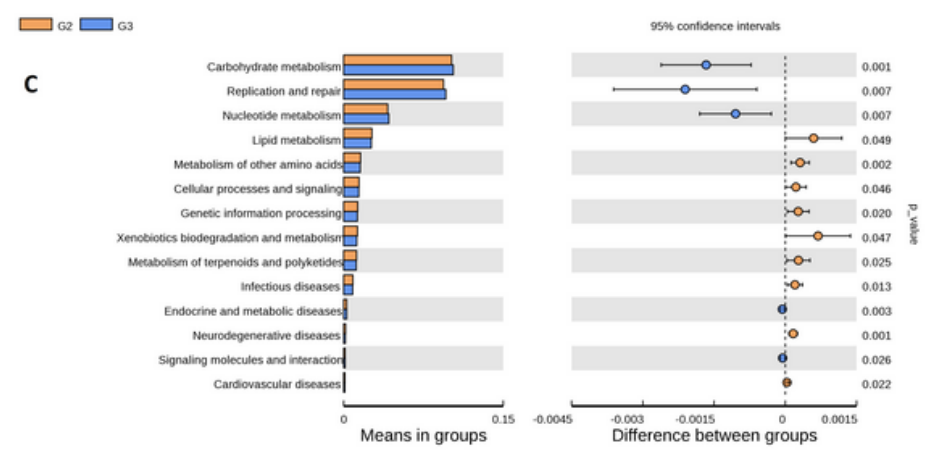
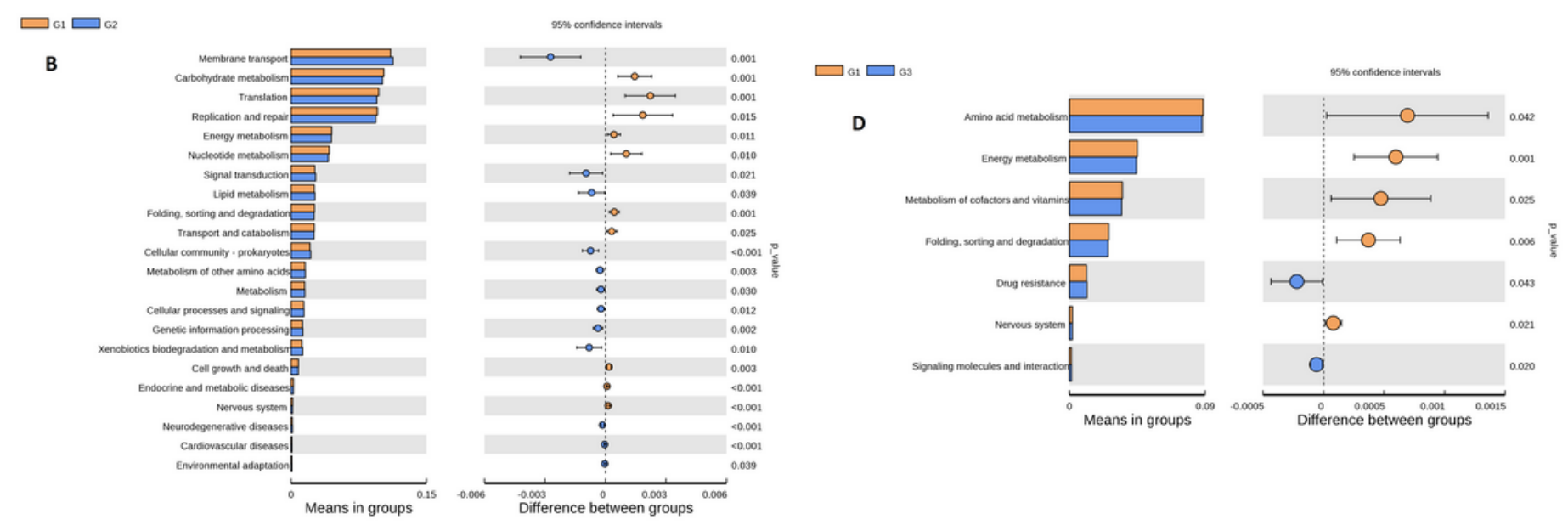

Figure 3

Heatmap of Tax4fun analysis showed important KEGG pathways during pregnancy (A). G1, G2, and G3 stand for early, middle and late pregnancy, respectively. The differentially expressed function prediction identified by paied-T tests between G1 and G2 (B) , between G2 and G3 (C) and between G1 and G3 (D) .

\section{Supplementary Files}

This is a list of supplementary files associated with this preprint. Click to download.

- FigureS1.png

- FigureS2.png

- FigureS3.png

- Supplementaryfiles.pdf 